\title{
PODER LEGISLATIVO, LEIS E SAÚDE BUCAL DOS BRASILEIROS
}

\section{LEGISLATIVE POWER, LAWS AND BRAZILIANS ORAL HEALTH}

\author{
Nilton Penha* \\ Charone Senda" \\ Marcos Corvino*** \\ Sonia Groisman ${ }^{* \cdots *}$
}

\begin{abstract}
RESUMO
O objetivo do presente trabalho é revisar as atribuições do processo legislativo, enumerando e avaliando os projetos de lei e leis implementadas na região sudeste do Brasil, nos Estados de São Paulo, Rio de Janeiro e Minas Gerais; traçando um paralelo entre eles, além de comparar o número de leis de saúde versus o número de leis em saúde bucal, e confrontar esses dados com a condição de saúde bucal da população brasileira. Os resultados demonstraram menores números de leis em Saúde Bucal e grande acometimento da doença cárie na população; o ranking de leis nos Estados pesquisados evidencia a pouca relevância dada à saúde bucal. Conclui-se que existe a necessidade de um posicionamento do Poder Legislativo, dos cidadãos civis e dos profissionais de saúde bucal em prol de uma melhoria dessa saúde e para se conseguir uma efetiva construção de cidadania.

DESCRITORES: Leis - Poder legislativo • Legislação sanitária • Saúde bucal • Políticas públicas de saúde

\section{ABSTRACT}

The aim of the present study is to evaluate legislative attributions, to count and evaluate the laws and implemented ones in the southwest of Brazil, in the States of São Paulo, Rio de Janeiro and Minas Gerais, comparing the number of health laws versus oral health laws and the number of congressmen as well as the population oral health. It was observed less oral health laws in comparison to general health ones and a poor oral health. The ranking between congressmen and the oral health laws is very low, indicating less relevance given to oral health. This study concluded that there is a need of the Legislative Power, dentists, and citizens in order to better the oral health and get the citizen construction.
\end{abstract}

DESCRIPTORS: Laws • Legislative power • Legislation, health • Oral health • Health public policy

\footnotetext{
* Cirurgião-Dentista, Especialista em Saúde Coletiva da Faculdade de Odontologia da UFRJ

** Doutoranda da Faculdade de Odontologia da USP-Bauru

*** Prof. Associado do Instituto da Saúde da Comunidade da Universidade Federal Fluminense

**** Professora Associada da Faculdade de Odontologia da Universidade Federal do Rio de Janeiro
} 
PENHA N

SENDA C

CORVINO M

GROISMAN $S$

PODER

LEGISLATIVO,

LEIS E SAÚDE

BUCAL DOS

BRASILEIROS

246

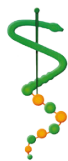

REV, ODONTOL,

UNIV. CID, SÃO

PAULO

$2011 ; 23(3): 245-$

52 , SET-DEZ

\section{INTRODUÇÃO E REVISÃO}

Segundo Vogel et al. ${ }^{1}$ (2005), democracia é uma palavra de origem grega que significa "poder do povo", (sendo "demos" povo e "cratos" poder). Na Grécia, durante o século IV A.C. o poder era exercido por uma assembleia de cidadãos, a quem competia elaborar as principais normas da vida em comunidade e decidir as questões de interesse comum. Contudo, nem todos os membros da sociedade podiam participar, sendo que destes estavam excluídos as mulheres, os escravos e os estrangeiros.

De acordo com os mesmos autores Vogel et al. ${ }^{1}, 2005$, atualmente no Estado moderno, em função da complexidade das sociedades e do expressivo número de cidadãos habilitados a participar do processo democrático, a democracia é representativa, isto é, os cidadãos escolhem, por intermédio do voto, os representantes que irão decidir os assuntos públicos, tanto no âmbito do Poder Executivo (Presidente da República, Governador de Estado e Prefeito) quanto no Poder Legislativo (Senador, Deputado Federal, Deputado Estadual e Vereador).

O processo Legislativo Constitucional compreende a elaboração de emendas constitucionais, leis complementares, leis delegadas, medidas provisórias, decretos legislativos e resoluções. Segundo estimativas do Instituto Brasileiro de Geografia e Estatística (IBGE², 2009), em 2009 o Brasil possuía 191,5 milhões de habitantes espaIhados pelas suas 27 unidades da federação e 5.565 municípios. As três unidades da federação da Região Sudeste que possuem as maiores economias, populações e quantidade de deputados, além de concentrarem cerca de $40 \%$ da população brasileira são: São Paulo, Rio de Janeiro e Minas Gerais.

Para chegar aos novos resultados populacionais, o IBGE$^{2}$ (2009) empregou uma metodologia de conciliação censitária combinada com o Método das Componentes Demográficas, uma ferramenta demográfica que visa obter as estruturas esperadas por sexo e idade das populações nos censos, à luz da dinâmica demográfica do país, procurando obter coerência entre os censos e contagens dos anos
1980, 1991, 1996, 2000 e 2007.

Os problemas bucais no Brasil ainda são considerados bastante prevalentes, constituindo-se em problemas de saúde pública com graves consequências sociais e econômicas. Dados nacionais demonstraram que o índice da doença cárie na dentição decídua (ceod) é de 1,1. Já para dentição permanente (CPO-D), esse índice atinge a média de 2,8 dentes aos 12 anos de idade. Tal resultado representa o alcance pelo Brasil da meta proposta pela OPAS/OMS ${ }^{3}$ (2000) de CPO-D menor que 3 em crianças até 12 anos. Os valores para faixa etária entre 15 a 19 anos elevam-se para 6,2. Na faixa etária adulta (35 a 44 anos) e entre idosos (65 a 74 anos) atingem níveis alarmantes, com valores médios de 20,1 e 27,8 respectivamente.

Resultados do Ministério da Saúde $\left(\right.$ Brasil $\left.^{4}, 2004\right)$ ressaltaram que mais de 2,5 milhões de adolescentes (13\%) nunca foram ao dentista. Entre a população adulta, quase 3\% nunca estiveram em um consultório odontológico e na população idosa esse número chega a $6 \%$. Em ambas as faixas etárias, a região Nordeste apresenta o maior índice de indivíduos que nunca foram ao dentista e a região Sul, os melhores valores relativos ao acesso a serviços odontológicos. A análise das estimativas do Projeto SB Brasil (Brasil ${ }^{4}$, 2004) revela que o declínio da cárie dentária na população infantil está ocorrendo de forma desigual na população brasileira, uma vez que existem disparidades relacionadas ao acesso a serviços.

Gonçalves et al. ${ }^{5}$ (2009) realizaram um estudo no município de Paraíba do Sul (Rio de Janeiro) em que o índice da doença cárie encontrado na dentição decídua (ceod) foi de 1,2. Já para dentição permanente (CPO-D) esse índice atingiu a média de 2,9 dentes aos 12 anos de idade. Para a faixa etária entre 15 a 19 anos, os valores elevaram-se para 5,9; na faixa etária adulta (35 a 44 anos) e entre idosos (65 a 74 anos) os valores atingiram níveis médios de 15,2 e 24,9 respectivamente. Os índices de doença cárie nesse município estão bem próximos aos valores encontrados no SB Brasil em crianças, porém com valores menores somente em adolescentes, adultos e idosos como descritos no Quadro I. 


\begin{tabular}{|c|c|c|}
\hline Faixa etária & População Paraíba do Sul & SB-BRASIL \\
\hline $0-4$ anos & 1.2 & 1.1 \\
\hline 12 anos & 2.9 & 2.8 \\
\hline $15-19$ anos & 5.90 & 6.2 \\
\hline $34-45$ anos & 15.2 & 20.1 \\
\hline $65-74$ anos & 24.9 & 27.8 \\
\hline
\end{tabular}

Quadro I - Número de escolares examinados (n), a população atendida pela estratégia do Programa de Saúde da Família (PSF) do Município de Paraíba do Sul (RJ).

Fonte: GONÇALVES et. al. ${ }^{5}(2009)$

\section{(Brasil $\left.{ }^{4}, 2004\right)$}

As doenças bucais têm sido relatadas como fatores de risco para doenças sistêmicas. Figueredo ${ }^{6}$ (2003) demonstrou a associação de doenças sistêmicas com a doença periodontal. Dentre essas doenças ou agravos, são considerados como fatores de risco para a doença periodontal o infarto agudo do miocárdio (IAM), acidentes vasculares cerebrais, crianças prematuras e de baixo peso, diabetes mellitus, infecções pulmonares crônicas, piora no quadro da evolução de pacientes renais crônicos e o fumo.

Para Campos et al. ${ }^{7}$ (2000), a mastigação é considerada um fator importante para a boa nutrição do idoso (Cormack $^{8}$, 1998, Nagao ${ }^{9}$, 1992). Com o grande número de dentes perdidos, o idoso tem sua capacidade mastigatória alterada, interferindo no comportamento inicial do processo digestório (Nogués ${ }^{10}, 1995$, Hayfli$\left.\mathrm{ck}^{11}, 1996\right)$.

Estudos demonstraram que indivíduos que utilizam dentaduras mastigam 75 a $85 \%$ menos que aqueles que possuem dentes naturais, levando a uma diminuição do consumo de carnes, frutas e vegetais, Tal fato aponta para o consumo inadequado de ferro e vitaminas, o que é prejudicial à saúde geral do idoso (Shu$\left.\operatorname{man}^{12}, 1998\right)$.

Novas medidas tomadas pelo governo com o intuito de diminuir a incidência dos acidentes de trânsito ("Lei Seca", programas de incentivo ao uso do cinto de segurança) têm surtido efeito, acarretando a diminuição da ocorrência de trauma de face (Brasileiro ${ }^{13}$, 2005). Outras medidas governamentais visando à inibição de agressões físicas contra mulheres, como a "Lei Maria da Penha", têm contribuído também para diminuição das fraturas de face. (Nedel et al. ${ }^{14}, 2009$ ).

Outro exemplo é a Lei Estadual Anti-fumo, para impedir fumantes de consumirem cigarros, charutos, cachimbos ou cigarrilhas em ambientes fechados, e evitar que indivíduos que não desejem fumar sejam afetados, atuem como fumantes passivos e acabem prejudicando sua saúde geral e bucal por hábitos de outrem, pois a literatura já evidenciou o risco de tumores pulmonares em fumantes, assim como acidentes cardiovasculares. Além disso, o fumo é considerado um fator de risco para diversas doenças sistêmicas e também para a Doença Periodontal. (Feliciano $^{15}, 2004$, Bolzan Pion et al. $\left.{ }^{16}, 2006\right)$.

Portanto, o presente trabalho objetivou enumerar as leis de saúde geral, comparando-as com as leis de saúde bucal que estão vigentes e, também, o número de projetos de lei de saúde com o número de projetos de lei de saúde bucal dos últimos dez anos nos Estados de São Paulo, Rio de Janeiro e Minas Gerais, de modo a estabelecer um perfil de relevância da saúde bucal da população brasileira (Cabral ${ }^{17}$, 2008).

\section{METODOLOGIA}

Esta pesquisa utilizou metodologia exploratória, visando identificar as Leis e Projetos de Leis referentes à saúde geral e à saúde bucal nos Estados de São Paulo, Rio de Janeiro e Minas Gerais, nos últimos 10 anos, através de pesquisa em sites das Assembleias Legislativas dos respectivos Estados (ALMG ${ }^{18}, 2009$, ALERJ $^{19}, 2009$, $\mathrm{ALSP}^{20}, 2009$ ).

A pesquisa foi realizada através de palavras-chave, sendo que para qualificar as Leis e Projetos de Lei em relação à saúde
PENHA N

SENDA C

CORVINOM

GROISMAN S

PODER

LEGISLATIVO,

LEIS E SAÚdE

BUCAL DOS

BRASILEIROS

247

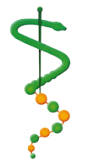

REV. ODONTOL.

UNIV, CID, SÃO

PAULO

2011; 23(3): 245 -

52, SET-DEZ 
PENHA N

SENDA C

CORVINO M

GROISMAN S

PODER

LEGISLATIVO,

LEIS E SAÚdE

BUCAL DOS

BRASILEIROS

\section{8}

REV, ODONTOL

UNIV, C I D, SÃO

PAULO

2011; 23(3): 245 -

52, SET-DEZ geral utilizou-se apenas a palavra "saúde" e para qualificar as Leis e Projetos de Lei em relação à saúde bucal, utilizaram-se as palavras "odontologia", "bucal", "dentista", "fluoretação", "doenças cárie" e "periodontal".

Foram excluídos as Leis e Projetos de Lei que se apresentaram repetidos e apareciam nas duas pesquisas, tanto na relação de saúde geral quanto na relação de saúde bucal, pois na justificativa para uma Lei ou Projeto de Lei as palavras apareceram repetidas, como, por exemplo, "saúde" e "bucal"; quando tal (is) palavra (s) foi (ram) encontrada(s) nas duas pesquisas, foram contabilizadas para lei ou projeto de lei em saúde bucal.

\section{RESULTADOS E DISCUSSÃO}

O Brasil se caracteriza atualmente como um país democrata, onde, para se assumir cargos públicos do Poder Legislativo (Vereadores, Deputados Estaduais e Federais) é necessário haver uma eleição através do voto. Tais representantes criam projetos de lei que devem passar pelas comissões referentes aos assuntos relacionados ao determinado projeto que, se aprovado, é encaminhado ao Poder Executivo (Prefeito, Governador e Presidente da República). Caso seja sancionado pelo Poder Executivo, tal projeto de lei se torna lei, seja ela Municipal, Estadual ou Federal (Vogel et al. $\left.{ }^{1}, 2005\right)$. O Gráfico I demonstra o número de Deputados Estaduais e Federais nos Estados de São Paulo, Rio de Janeiro e Minas Gerais. Os resultados deste estudo evidenciaram a relação entre o

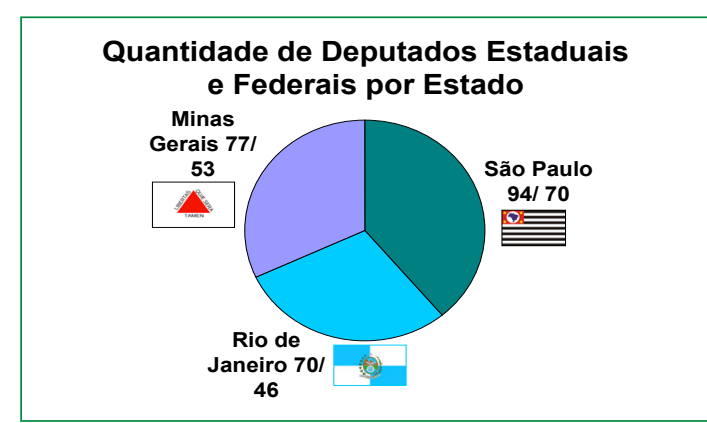

Gráfico I - Número total de Deputados nos Estados de São Paulo, Rio de Janeiro e Minas Gerais.

Fonte: Sites das Assembleias Legislativas de cada Estado (www. al.sp.gov.br; www.alerj.rj.gov.br ; www.almg.gov.br e www. camara.gov.br). número de Deputados Federais e Estaduais para os três Estados pesquisados, visto que eles são os responsáveis pela formulação de projetos de leis e, por consequência, leis federais e estaduais.

No que tange ao quantitativo de Projetos de Lei em Saúde Bucal (P.L.'s) que tramitam nas Assembleias Legislativas Estaduais de 1999 até 2009, observa-se que nos últimos 10 anos, São Paulo aparece em primeiro lugar com 44 P.L.'s, em segundo Minas Gerais com 21 P.L.'s e em terceiro lugar o Rio de Janeiro com 14 P.L.'s. Dentre esses projetos de lei, podemos citar o de número 1804 do ano de 2008 (Cabral ${ }^{17}, 2008$ ), o qual inclui a Ementa: "Determina a instalação de escovódromos nos restaurantes populares localizados no Estado do Rio de Janeiro" e está contabilizada como um dos 14 Projetos de Lei em tramitação no Estado do Rio de Janeiro, exemplificado no Gráfico II.

De acordo com os sites das Assembleias Legislativas (ALMG ${ }^{18,}$ 2009, ALERJ ${ }^{19}$, 2009, ALSP20, 2009), no dia 18 de abril de 1958 a lei paulista de número 4.687 foi a pioneira em Odontologia e do universo de 3771 leis relativas à saúde geral, até hoje apenas 24 possuem seu tema em Saúde Bucal. Proporcionalmente temos a razão de 1 (uma) para 157 (cento e cinquenta e sete), ou seja, para cada 157 leis em saúde geral sancionadas é criada 1 lei em saúde bucal, ou, por outro ângulo, o número total de leis em saúde geral é de 157 vezes superior ao número total de leis em saúde bucal.

Gão Paulo Rio de J aneiro Minas Gerais

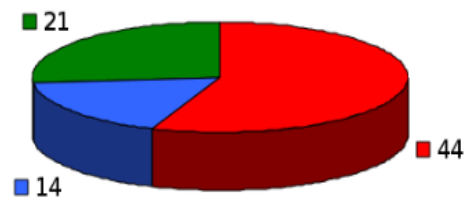

Gráfico II - Quantidade de Projetos de Lei em Saúde Bucal que Tramitam nas Assembleias Legislativas Estaduais de 1999 até 2009.

Fonte: Sites das Assembleias Legislativas de cada Estado (www. al.sp.gov.br; www.alerj.rj.gov.br; www.almg.gov.br). 


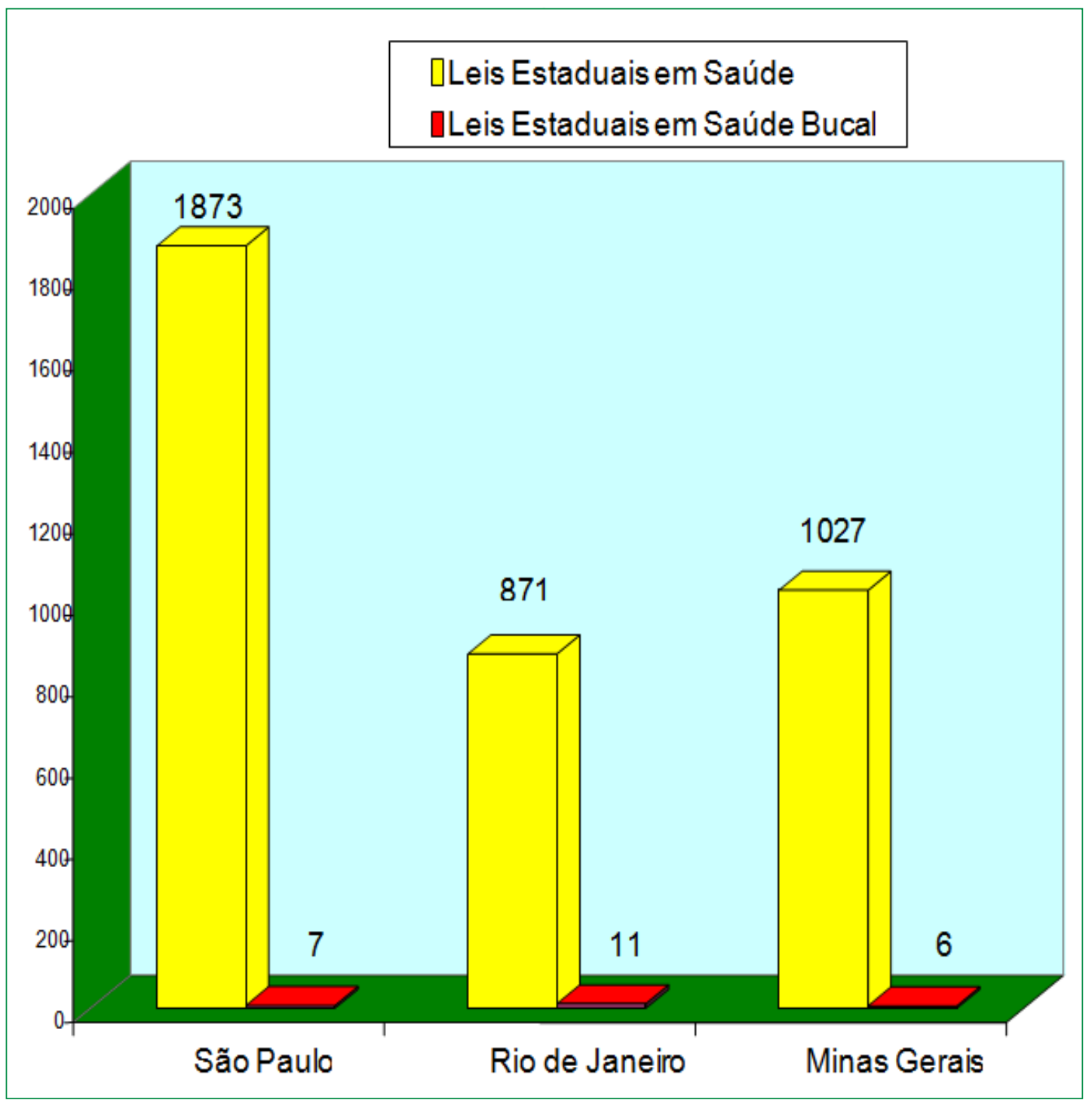

Gráfico III - Quantidade de Leis Estaduais em Saúde e Saúde Bucal

Fonte: Sites das Assembleias Legislativas de cada Estado (www.al.sp.gov.br; www.alerj.rj.gov.br; www.almg.gov.br).

Quando se comparam o número de Leis de Saúde Geral (LSG) e o número de Leis em Saúde Bucal (LSB), observa-se que o Estado do Rio de Janeiro possui a melhor proporção entre os três Estados (LSG 871/ LSB 11), seguido por Minas Gerais (LSG 1027/ LSB 06) e São Paulo (LSG 1873/ LSB 07). Esses dados apontam que, no Rio de Janeiro, para cada 79 Leis em Saúde Geral é criada uma Lei em Saúde Bucal, em Minas Gerais esse número aumenta e para cada 171 Leis em Saúde Geral é criada uma Lei em Saúde Bucal e em São Paulo para cada 267 Leis em Saúde Geral é criada uma Lei em Saúde Bucal (Gráfico III).

A relação direta entre Leis e saúde, levando ao bem-estar público, pode ser exemplificada pela lei federal denominada "Lei Seca" que não permite, no terri- tório nacional, o consumo de álcool aos cidadãos que dirigem veículos automotores. Isso tem acarretado a diminuição de acidentes automobilísticos, traumas gerais e faciais (Coelho Júnior et al. ${ }^{21}, 2008$ ). Dentro dessa perspectiva, uma lei de trânsito pode melhorar a saúde, assim como uma Lei de Saúde Bucal específica pode melhorar a saúde geral. Outro exemplo é a Lei Estadual Antifumo, que impede fumantes de consumirem cigarros, charutos, cachimbos ou cigarrilhas em ambientes fechados, evitando que indivíduos que não desejem fumar se tornem fumantes passivos, prejudicando sua saúde geral e bucal pelos hábitos de outras pessoas, pois a literatura já evidenciou o risco de tumores pulmonares em fumantes, sejam fumantes ativos ou passivos, assim como

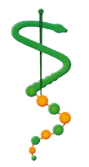

REV, ODONTOL.

UNIV, CID, SÃO PAULO 
PENHA N

SENDA C

CORVINO M

GROISMAN $S$

PODER

LEGISLATIVO,

LEIS E SAÚDE:

BUCAL DOS

BRASILEIROS
I SSN 1983-5183

acidentes cardiovasculares. Além disso, o fumo também diminui a resposta do hospedeiro ao agente agressor, agravando ou dificultando o tratamento periodontal (Bolzan Pion et al. ${ }^{16}$, 2006).

Observou-se, também, a relação direta entre doenças, patologias e agravos cardiovasculares e doenças periodontais, fazendo-se uma associação entre fumo, doenças cardíacas e periodontais, patologias e agravos (Feliciano ${ }^{15}$, 2004). Sob essa perspectiva, os resultados apresentados no presente trabalho constataram que a proporção de leis de saúde geral é maior do que as leis de saúde bucal dentro desses três Estados. Tais dados são evidenciados no SB Brasil5, em que $13 \%$ da população brasileira nunca foi ao dentista, além de haver na população jovem e adulta um alto índice de CPO-D, com um grande número de dentes perdidos e necessidade de serem confeccionadas próteses dentárias (Brasil ${ }^{4}, 2004$, Gonçalves et al. ${ }^{5}, 2009$ ).

Em relação à quantidade de Deputados nos Estados de São Paulo, Rio de Janeiro e
Minas Gerais correlacionada com a quantidade de Leis Estaduais em Saúde Bucal, foi observado que em São Paulo, com 94 Deputados Estaduais, foram criadas 7 leis em saúde bucal, em Minas Gerais com 77 Deputados Estaduais criaram-se 6 leis em saúde bucal e no Rio de Janeiro, com 70 Deputados Estaduais, foram feitas 11 leis em saúde bucal (Gráfico IV).

Quando se retrata o número de deputados e Leis de Saúde Bucal por Estado, fica lícito dizer que precisa existir maior conscientização dos representantes do poder legislativo, sejam eles Estaduais e/ ou Federais. E por parte dos cidadãos civis e dos profissionais de saúde bucal, os cirurgiões-dentistas, o intuito de empoderar representantes legais dotados de conhecimento sobre a relevância de Leis em prol da saúde bucal, que trará, por conseguinte, melhorias nas condições de saúde geral e na qualidade de vida dos brasileiros.

\section{CONCLUSÕES}

O número de leis de Saúde Bucal é

Gráfico IV - Quantidade de Deputados pelos Estados de São Paulo, Rio de Janeiro e Minas Gerais e Quantidade de Leis Estaduais em Saúde Bucal.

Fonte: Sites das Assembleias Legislativas de Cada Estado (www.al.sp.gov.br; www.alerj.rj.gov.br; www.almg.gov.br).

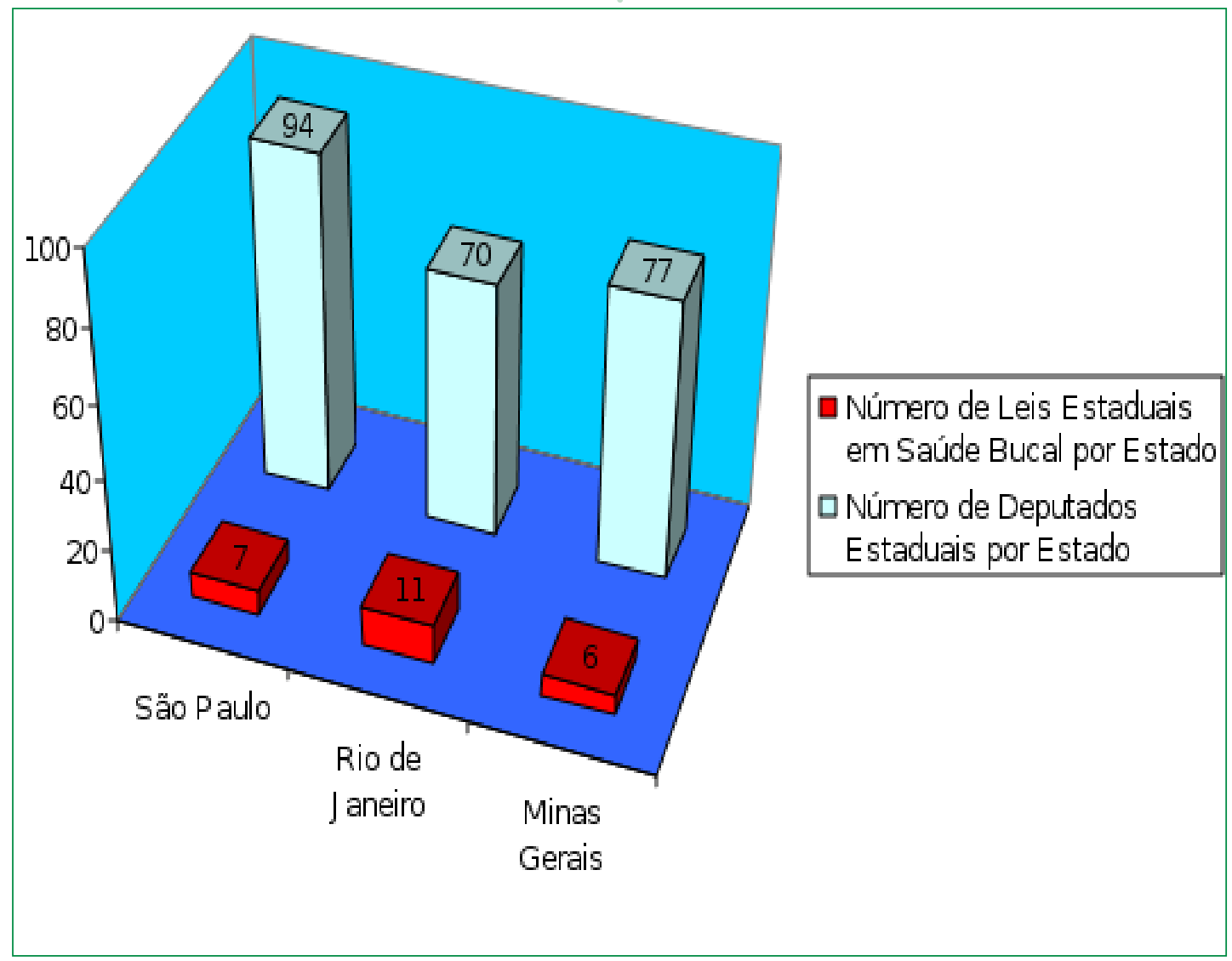


bem menor do que as de Saúde Geral.

As Leis por Estado evidenciaram pouca relevância referente aos dados dos levantamentos realizados em saúde bucal.
Existe a necessidade de um posicionamento do Poder Legislativo e dos cidadãos brasileiros em prol de uma melhor saúde bucal para a efetiva construção da cidadania.

\section{REFERÊNCIAS}

1. Vogel L, Martins R, Xavier R. O poder legislativo no Brasil: um Estado republicano, democrático e representativo2005. Disponível em: http://apache.camara.gov.br/ portal/arquivos/Camara/internet/conheca/poderlegislativo.pdf.

2. IBGE. Instituto Brasileiro de Geografia e Estatística. 2009 [Acesso em 2009 2]; Disponível em: http://www.ibge.gov.br/home/.

3. OPAS/OMS. Organização Pan-Americana da Saúde. Saúde bucal. 2000. Disponível em: http://www.opas.org.br/sistema/fotos/bucal.pdf.

4. Brasil. Ministério da Saúde. Secretaria de Atenção à Saúde. Departamento de Atenção Básica. Projeto SB Brasil 2003: condições de saúde bucal da população brasileira 2002-2003: resultados principais. Brasília: Ministério da Saúde; 2004. Disponível em: http://dab.saude.gov.br/cnsb/publicacoes.php.

5. Gonçalves RM, Corvino MPF, Groisman S, Olival ARBd, Milanos ER, Toledo E. Prevalência da doença cárie em município do estado do Rio de Janeiro. Perionews 2009.

6. Figueredo CM. Odontologia em alto nível. 2003. Disponível em: http://www.periodontiamedica.com.br/entrevista-concedida-ao-blog-brazilian-dentists/.

7. Campos MTFdS, Monteiro JBR, Ornelas APRdC. Fatores que afetam o consumo alimentar e a nutrição do idoso. Rev Nutr 2000 13(3):157-65.

8. Cormack E. A saúde oral do idoso. 1998. Disponível em: www.odontologia.com.br/ artigos/geriatria.html.

9. Nagao M. The effects of aging on mastication. Nutr Rev 1992 Dec;50(12):434-7.

10. Nogués R. Factors que afectan la ingesta de nutrientes en el anciano y que condicionan su correcta nutrición. Nutrición Clínica 1995 15(2):39-44.

11. Hayflick L. Como e porque envelhecemos. Rio de Janeiro: Campus; 1996.

12. Shuman J. Nutrição no envelhecimento. In: Mahan L, Stump S, editors. Alimentos, nutrição e dietoterapia. 9.ed. ed. São Paulo: Roca; 1998. p. 293-312.

13. Brasileiro BF. Prevalência, tratamento e complicações dos casos de trauma facial atendidos pela FOP - Unicamp de abril de 1999 a março de 2004 [Mestrado]. Piracicaba, SP Universidade Estadual de Campinas. Faculdade de Odontologia de Piracicaba; 2005.

14. Nedel A, Nedel F, Conceição L, Silva R, Lund R. Prevalência de lesões corporais em região oro-facial registrados no Instituto Médico Legal de Pelotas/RS2009. Disponível em: http://www.ufpel.edu.br/cic/2009/cd/pdf/CS/CS_01106.pdf.

15. Feliciano C. A doença periodontal como fator de risco nas enfermidades cardiovasculares [Monografia]. Rio de Janeiro: Unigranrio; 2004.

16. Bolzan Pion FL, Araujo MWB, Feres M, Cortelli SC. Condição periodontal de um subgrupo populacional do município de Guarulhos, SP. Rev bras epidemiol 2006 Sept.;9(3):335-45.

PENHA N

SENDA C

CORVINO M

GROISMAN S

PODER

LEGISLATIVO,

LEIS E SAÚdE

BUCAL DOS

BRASILEIROS

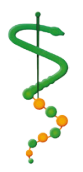

REV. ODONTOL.

UNIV, CID, SÃO

PAULO

2011; 23(3): 245 -

52, SET-DEZ 
PENHA N

SENDA C CORVINO M

GROISMAN S

PODER

LEGISLATIVO,

LEIS E SAÚDE

BUCAL DOS

BRASILEIROS

- 252

17. Cabral W. Projeto de Lei no 1804/2008: Ementa: determina a instalação de escovódromos nos restaurantes populares localizados no estado do Rio de Janeiro. 2008. Disponível em: http://alerjln1.alerj.rj.gov.br/scpro0711.nsf/18c1dd68f96be3e78325 66ec0018d833/baca559dc1 ce52c4832574e30072b52a.

18. ALMG. Assembléia de Minas: poder e voz do cidadão. 2009. Disponível em: http:// www.almg.gov.br/home/index.html.

19. ALERJ. Assembléia Legislativa do Estado do Rio de Janeiro. 2009. Disponível em: http://www.alerj.rj.gov.br/.

20. ALSP. Assembléia Legislativa do Estado de São Paulo. 2009. Disponível em: http:// www.al.sp.gov.br/portal/site/Internet/.

21. Coelho Júnior RG, Carvalho MRMS, Aquino JEP, Fernandes JCR, Brandão FH, Pereira $\mathrm{SH}$, et al. Estudo epidemiológico de trauma nasal em um ambulatório otorrinolaringológico da zona sul de São Paulo. Arq int otorrinolaringol (Impr) 2008 jul.-set.;12(3):356-61.

Recebido em: 24/09/2010

Aceito em: 28/03/2011 